\title{
Effects of Osteopathic Treatment on Postural Equilibrium Evaluated through a Stabilometric Platform: A Randomized and Controlled Study
}

\author{
Andrea Buscemi ${ }^{1}$, Marcello Cannatella ${ }^{2}$, Paolo Lutrario ${ }^{2}$, Alessandro Rapisarda ${ }^{1}$, \\ Giulia Di Gregorio ${ }^{3}$ and Marinella Coco ${ }^{4, *}$ \\ 1 Department of Research, Center Studies of Osteopathy, Catania 95129, Italy; andreabusemi@virgilio.it (A.B.); \\ arapisarda@csdoi.it (A.R.) \\ 2 Orthopedic Study “Balduina”, Roma 00118, Italy; abuscemi@csdoi.it (M.C.); ritanicotra@gmail.com (P.L.) \\ 3 Department of Education Sciences, University of Study of Catania, Catania 95100, Italy; \\ giuliadigregor@gmail.com \\ 4 Horus Cooperative Social, Ragusa 97100, Italy \\ * Correspondence: marinella.coco@gmail.com; Tel.: +39-33-8153-6579; Fax: +39-095-604-125
}

Academic Editors: Paul Stapley and Giuseppe Musumeci

Received: 6 April 2017; Accepted: 17 May 2017; Published: 24 May 2017

\begin{abstract}
Equilibrium is a very complex function and involves many systems, including the somatic-sensory, vestibular and visual systems. The condition of balance is maintained until the gravity center falls into the perimeter of support, defined as the ground level of the support base. Equilibrium loss is at the root of the risk of falling down, and represents a public health issue associated with remarkable costs for hospitalization, risk of residual disability and death for the elderly. Osteopathy can be a good ally that allows the human body to oppose gravitational force in a functional and ergonomic way. Osteopathy represents a medical approach complementary to healthcare and could be a non-invasive approach to improve and support traditional medicine in different physiological and pathological conditions. The aim of this study is to evaluate the cause-effect relationship between Manipulative Osteopathic Treatment (OMT) and its effects on equilibrium through stabilometric examinations. The 63 chosen subjects, including 23 females and 40 males, were between 18 and 45 years old, with a mean age of 29 . Subjects were randomly divided into two groups: Manipulative Osteopathic Treatment (OMT) and Non Touch group (NON TOUCH). We can confirm that the tonic-postural response was more marked in the OMT group, with a statistic relevance both for the ellipse surface and the tangle length, with better stability and consequent energy saving in treated subjects. There were not statistically relevant variations for the NON TOUCH group. Therefore, it seems that Manipulative Osteopathic Treatment (OMT) influences stabilometry. However, posture influence due to Manipulative Osteopathic Treatment (OMT) should be evaluated with short- and long-term follow-ups, and with a larger sample size to test the administration of osteopathic treatments, in order to define an adequate work plan time period to prevent equilibrium loss.
\end{abstract}

Keywords: posture; equilibrium; osteopathic manipulative treatment

\section{Introduction}

Equilibrium is a term that refers to an optimal condition in which a subject assumes a posture or a series of postures that are ideal for the adaptation to the ambiant situation he finds himself in at a precise moment. Equilibrium is a very complex function and involves many systems, including the somatic-sensory, vestibular and visual systems [1]. Every one of these systems has proprioceptive and exteroceptive receptors; every component interacts amongst themselves with tonic-postural activity [2]. 
Equilibrium can be "static" in the absence of voluntary movements, or "dynamic" if it concerns a motor situation.

The condition of balance is maintained until the gravity center falls into the perimeter of support, defined as the ground level of the support base.

Outside the border of the perimeter of support, static attitudes are no longer possible; instead, movement is necessary to further enlarge the support base or to modify the projection of the center of gravity.

Baron affirms that "standing in static condition, the body is never immobile; it swings permanently following particular and complex rhythms, of which wideness and frequency depends on the information received from the different sensory-motors systems, which position and maintain the gravity center inside the polygon of sustain of upright man" [3].

Therefore, posture can be defined as the totality of commands that postural-tonic activity chooses in a precise time and place to adapt and contrast to gravitational force both during movement and static conditions.

Equilibrium loss is at the base of the risk of falling down, which represents a public health issue associated with remarkable costs for hospitalization, risk of residual disability and death for the elderly. For this reason, an increase in physical activity and interventions focused on resistance, muscular flexibility, and equilibrium are strongly recommended both in young people, to improve sports performance, and elderly people [4,5].

The human body, like all perfect mechanisms, needs the right calibration between its devices to work at its best and to counteract the constant action of gravity. Every one of us is in a constant fight with gravity, and everyone has his method to confront it; this is true both for those who spend hours working in an office and for those who are on the starting blocks ready to run the $100 \mathrm{~m}$ dash in the Olympics.

Posture and deambulation studies highlight that weight distribution on a plantar surface can change in relation to a subject's structural characteristics. It can be influenced by alterations of different bone segments (tarsus, metatarsus, tibia, femur, hip bone).

Osteopathy, developed by Andrew Taylor Still (1828-1917), is a complementary integrative medicine which is based on the fundamental principles of integration between mind, body and spirit. As Still affirms, "Osteopathy is based on perfection in Nature's work. When all the parts of the body are put in order we are healthy, if they are not, the consequence is a disease. When the parts are readjusted disease gives way to health; the work of an osteopath consists of readjusting the body bringing him from abnormality to normality, after that, the abnormal condition gives way to the normal condition and health is the result" [6].

Osteopathy can be a good ally to help the human body to oppose gravitational force in a functional and ergonomic way. Osteopathy represents a medical approach complementary to healthcare and could be a non-invasive approach to improve and support traditional medicine in different physiological and pathological conditions.

The aim of this study is to evaluate the cause-effect relationship between Manual Osteopathic Treatment (OMT) [1] and its effects on equilibrium analyzed through stabilometric examinations.

We did not choose to utilize single techniques [7,8], but rather to intervene following a specific therapeutic process individually adapted to each patient with respect to osteopathy principles [9].

\section{Materials and Methods}

\subsection{Participants}

This was a randomized and controlled study. Participant selection was conducted first through advertising posters and then by direct contact, by recruiting familiars, work acquaintances or athletes belonging to gyms and sport groups. One hundred volunteers were recruited for this study.

The following exclusion criteria were defined as follows [10]: 
(1) Vestibular problems (vertigo, dizziness, Ménière Syndrome).

(2) Aged less than 18 or more than 45 years.

(3) Bone fractures of lower limbs, hip bones, or vertebral column.

(4) Muscular or ligament wounds that are recent or in course.

(5) Surgery (basing on the specific case).

(6) Degenerative problems (malformations, tumors).

(7) Prosthesis and amputations.

(8) Hearing problems.

(9) Neurological pathology.

Age restrictions were due to a bureaucratic factor linked with policy consensus (minimum of 18 years of age) and also because of clinic reasons; from maturity to aging we can see a progressive and significant impairment of balance (maximum of 45 years of age) [11].

Out of 100 subjects we recruited 63; 37 subjects were rejected according to the exclusion criteria.

The 63 recruited subjects included 23 females and 40 males aging between 18 and 45 years, with a mean age of 29 years.

Subjects were randomly divided into two groups: a Manipulative Osteopathic Treatment (OMT) group and a group without treatment (NON TOUCH). The subjects were not aware of which group they were in.

We established some work days, freely chosen by the subjects, during which stabilometric tests were performed.

The 30 subjects of the OMT group were tested on Monday, whereas the 33 subjects of the NON TOUCH group were tested on Thursday.

\subsection{Methods}

Experimental protocol is listed as follows (Figure 1):

(1) Both groups, the OMT group and the NON TOUCH group, were subjected to the stabilometric test.

(2) The sample was split into two groups; the OMT group was subjected to osteopathic manipulative treatment according to the Black Box Design [12], while the NON TOUCH Group waited for 30 min lying down or sitting. The Black Box Design incorporates all the tests and techniques from which the therapist may draw to assign the manipulative treatment.

(3) Both groups, the OMT group and the NON TOUCH group, were subjected again to the stabilometric exam.

\section{EXPERIMENTAL PROTOCOL}

OMT GROUP

treatment

TESTS STABILOMETRY
TESTS STABILOMETRY

NON TOUCH GROUP

wait relaxed

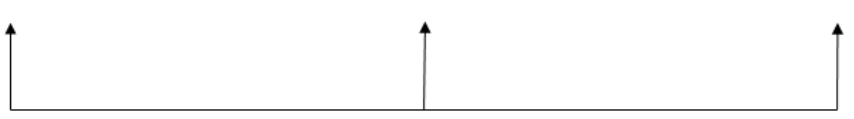

Figure 1. Experimental protocol. 
A certain level of blindness was present between the collaborators; the osteopath did not know the stabilometric test results evaluated by the technician and the technician, on his side, did not know which group the subject he examined was part of.

Subjects were managed by a secretary and were sorted between the waiting room, the stabilometric exam room and the osteopathic ambulatory.

Another level of blindness in the procedure was that the subjects did not know what the study was evaluating, in order to avoid different efforts in balance tests.

\subsection{Black Box Design}

\section{Stabilometric Exam}

Stabilometry evaluates the oscillations in erect posture in conditions of calmness and in absence of perturbation [13]. Stabilometric analysis provides data about the equilibrium of the examined subject; it reveals the oscillations that the body makes as an expression of balance-maintaining mechanisms. The graphic produced during the test is called "static posturography" as shown in Figure 2.

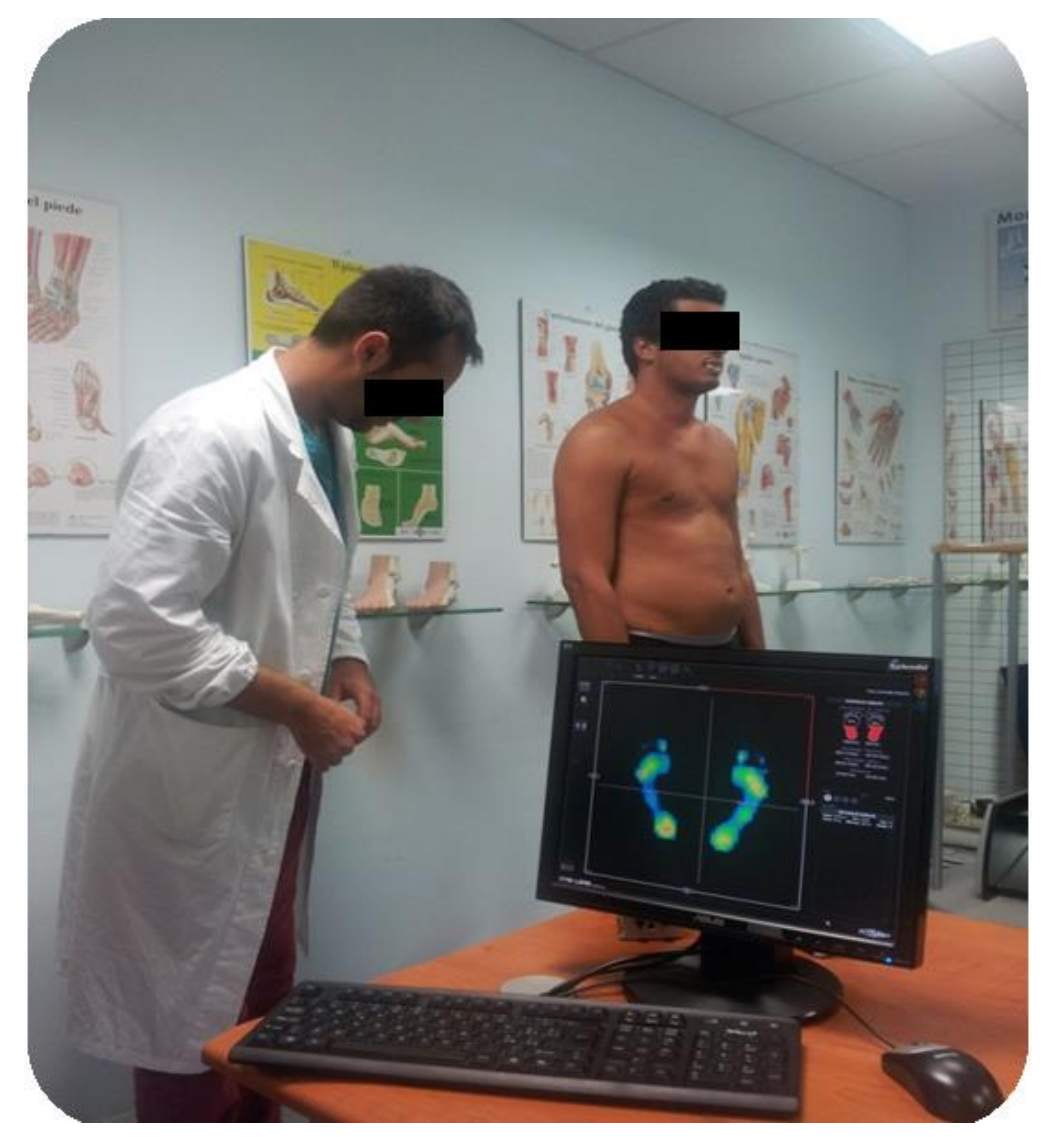

Figure 2. Example of a stabilometric test.

Static posturography describes and represents with a graphic the movements of the center of gravity of the person, in absence of voluntary movements; it also shows postural strategies adopted in order to maintain the erected station. The test consists of recording for $52 \mathrm{~s}$ the variation in weight charge, using torque sensors positioned on the inside of a foot board. After this, a software linked with the foot board extrapolates the data that characterize the movement of the center of gravity during the test. The exam also allows for a quantitative and qualitative evaluation of static postural control [14].

We used an EcoSanit ${ }^{\circledR}$ stabilometric foot board and StabiloPro2 software (version 2016, PROeREAL, Milano, Italy), using the same procedure for every subject: 
(1) Same feet position on the foot board.

(2) Without clothes and barefoot.

(3) Closed eyes (to neutralize visual component).

(4) Same environment conditions (light, temperature, daytime) $[15,16]$.

Keeping the eyes closed during erected station requires shifting one's attention to the vestibule and proprioceptive input in order to maintain balance; this is associated with an increase of body oscillations or with changes in body oscillations in healthy subjects [17].

\subsection{Statistical Analysis}

Data was collected and averaged, and then compared by using one-way repeated measures analysis of variance (ANOVA; Friedman test), followed by post-hoc Dunn's Multiple Comparison Test. The relationship between variables was analyzed with a linear regression. Significance was set at $p<0.05$ and all data are reported as means \pm standard deviation (SD). All analyses were performed using GraphPad Prism version 6.03 for Windows (GraphPad Software, Inc., La Jolla, CA USA).

\section{Results}

Analyzing the surface of the ellipses, we can see (Figures 2 and 3; Table 1) that the OMT group exhibited values in the range of $51.2 \mathrm{mmq}$ (square millimeters) pre- to 44.13 post-test, with a difference of $-17.1 \%$. This indicates a reduction of the oscillation surface, meaning a significant improvement. The decrease of this parameter causes the amplitude decrease to be directly proportional to a decrease in energy expenditure on the subject's part.

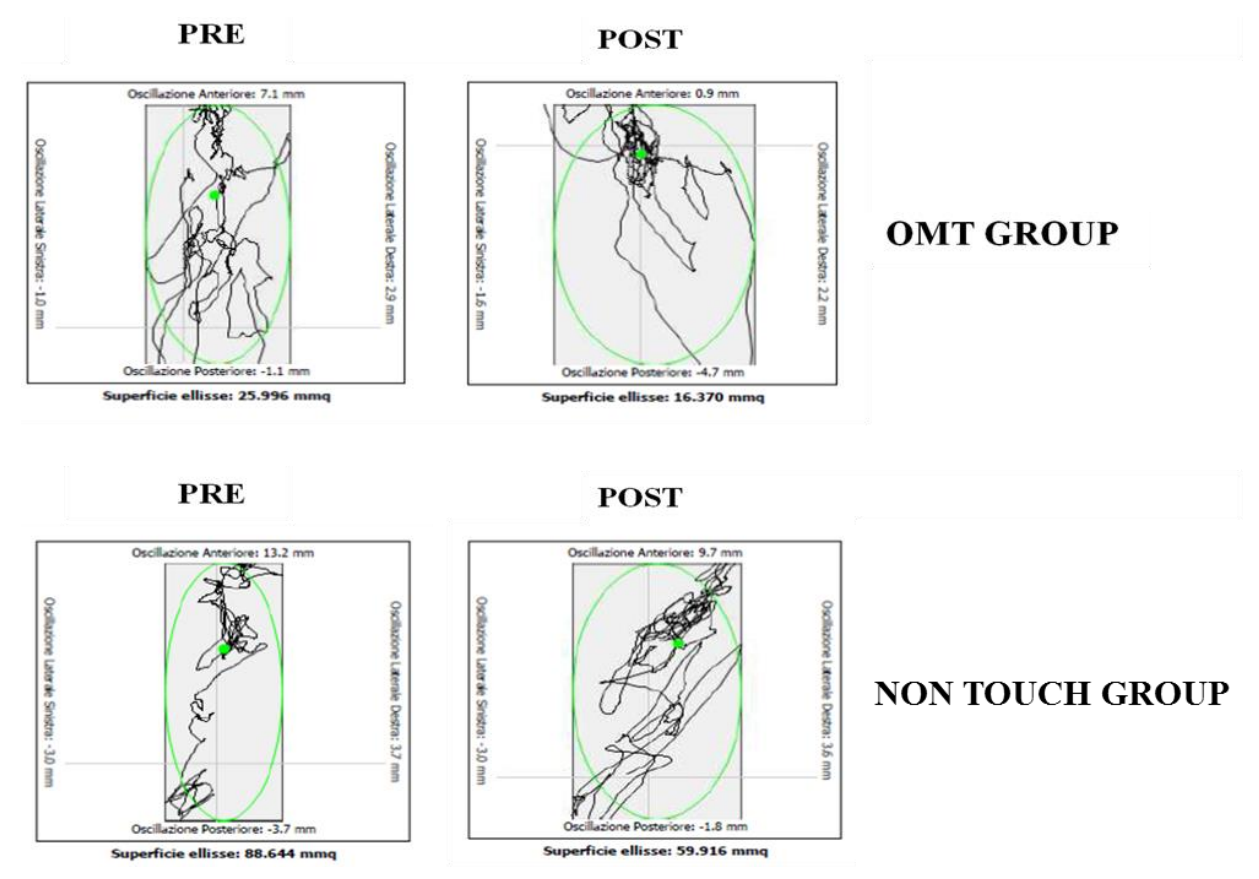

Figure 3. Results obtained from the stabilometric foot board from two subjects representative of the sample groups, Manipulative Osteopathic Treatment (OMT) and Non Touch group (NON TOUCH). 
Table 1. Values obtained from the stabilometric foot board from two subjects representative of the sample groups.

\begin{tabular}{ccccc}
\hline \multirow{2}{*}{ Values Stabilometric } & \multicolumn{2}{c}{ OMT GROUP } & \multicolumn{2}{c}{ NON TOUCH GROUP } \\
\cline { 2 - 5 } & PRE & POST & PRE & POST \\
\hline Duration of examination (s) & 52 & 52 & 52 & 52 \\
Mean center of gravity (mm) & $X: 0.8 ; Y: 4.2$ & $X: 0.0 ; Y:-0.1$ & $X: 0.3 ; Y: 7.5$ & $X: 1.1 ; Y: 6.0$ \\
Standard deviation X & 0.1 & 0.1 & 0.1 & 0.2 \\
Standard deviation Y & 0.2 & 0.1 & 0.5 & 0.3 \\
Distance of the tangle & 102.9 & 70.5 & 132.7 & 162.7 \\
Overall speed (mm/s) & 2.0 & 1.4 & 2.6 & 3.1 \\
Distance/Surface (LSF) & 4.0 & 4.3 & 1.5 & 2.7 \\
Speed/Shift (VFY) & 8.5 & 13.1 & 4.8 & 10.3 \\
Sway Area (mmq) & 33.0 & 30.1 & 102.8 & 87.4 \\
\hline
\end{tabular}

The NON TOUCH group, instead, exhibited values from the range of $63.25 \mathrm{mmq}$ pre- to $64.74 \mathrm{mmq}$ per second post-test, with a difference of $+1.5 \%$ (Figures 2 and 3; Table 1). This does not show a decrease in the amplitude of the oscillatory surface, indicating that there were no improvements.

The OMT group showed that the distance of the tangle values decreased from $119.55 \mathrm{~cm}$ pre- to $114.80 \mathrm{~cm}$ post-test; we can see a moderate decrease equal to $4 \%$. On the other hand, for the NON TOUCH group, values increased from $121.78 \mathrm{~cm}$ pre- to $147.81 \mathrm{~cm}$ post-test, with a more consistent increase equal to $+21 \%$ (Figures 2 and 3; Table 1).

\section{Discussion}

Stabilometry is a methodical discipline developed to evaluate balance capacity and relative energy expenditure.

The foot board was an important diagnostic aid that allowed us to evaluate the reactions of tonic-postural activity after the "administration" of an OMT.

In comparing the results obtained from the test on the two groups, we can assert that tonic-postural answers were more emphasized in the OMT group with a statistic relevance both for the ellipse amplitude and the tangle length, resulting in a better stability and consequently less energy expenditure in treated subjects. While there were not statistically relevant variations for the NON TOUCH group, OMT appears to influence stabilometry. However, the maintenance of influence in posture should be evaluated by short-term and long-term follow-ups, as well as the administration of a larger number of osteopathic treatments in order to define an adequate work plan time period to propose for the prevention of balance loss.

The small number of participants is a limitation of this study.

\section{Conclusions}

In conclusion, the use of the stability test for balance evaluation has been a valid and reliable method that has allowed us to evaluate the oscillations in erect posture in a calm state and in the absence of perturbation.

We can affirm the scientific relevance of this research, especially for athletic and post-rehabilitative patients. These positive results encourage us to confirm the notable and favorable use of osteopathic manipulative techniques for posture, with application perspectives both in injury prevention and the improvement of athletic performance.

Considering these results, it would be interesting to compare post-injury sportsmen and high level athletes, in order to evaluate the improvement range between pathological individuals, healthy individuals, and professional athletes.

Acknowledgments: Thanks to Doctor of Osteopathy (D.O.) Andrea Buscemi Responsible of Research and thesis Department Center Studies of Osteopathy (C.S.d.O.I.-Catania) as well as our supervisor, who laid the foundation 
of our study, providing us with guidelines to adopt for the development of this scientific research. We thank every person who took part in this study and had the patience and courtesy to respect the appointments. We express our gratitude to Orthopedic Technician Carlo Alberti who allowed us to use his study, his machinery, his professionalism and competence. Finally, a sincere thanks to Doctor of Osteopathy (D.O.) Alessandro Rapisarda, Director of Center Studies of Osteopathy (C.S.d.O.I) - Catania, who received us in his school, allowing us to conclude our course of study in Osteopathy. All sources of funding of the study should be disclosed. Please clearly indicate grants that you have received in support of your research work. Clearly state if you received funds for covering the costs to publish in open access.

Author Contributions: Marinella Coco and Andrea Buscemi conceived and designed the experiments; Marinella Coco and Andrea Buscemi performed the experiments; Marinella Coco analyzed the data; Marinella Coco, Andrea Buscemi, Marcello Cannatella, Paolo Lutrario, Alessandro Rapisarda, Giulia Di Gregorio contributed reagents/materials/analysis tools; Marinella Coco and Andrea Buscemi wrote the paper.

Conflicts of Interest: The authors declare no conflict of interest.

\section{References}

1. Ward, R.C. Glossary of ostheopatic terminology. In Foundation for Ostheopatic Medicine; Lippincott Williams and Wilkins: Baltimora, MD, USA, 2003; pp. 1229-1253.

2. Ceccaldi, A.; Favre, J.F. I Pivot Osteopatici; ISBN 8874492391.

3. Baron, J.B. Postural aspects of the post-concussional syndrome. Clin. Otolaryngol. Allied Sci. 1980, 5, $215-219$. [CrossRef] [PubMed]

4. Bergamin, M.; Gobbo, S.; Bullo, V.; Zanotto, T.; Vendramin, B.; Duregon, F.; Cugusi, L.; Camozzi, V.; Zaccaria, M.; Neunhaeuserer, D.; et al. Effects of a Pilates exercise program on muscle strength, postural control and body composition: Results from a pilot study in a group of post-menopausal women. Age 2015, 37, 118. [CrossRef] [PubMed]

5. Buscemi, A; Carbone, J.; Tacchi, M.; Buttafuoco, S.; Rapisarda, A.; Perciavalle, V.; Coco, M. Changes of urine $\mathrm{pH}$ after the compression of the fourth ventricle. Scienze e Ricerche 2014, 2, 122-126.

6. Still, A.T. Autobiografia; Mogni, S., Traduttore, S., Eds.; Castello Editore: Milan, Italy, 2000.

7. Alburquerque-Sendín, F.; Fernández-de-las-Peñas, C.; Santos-del-Rey, M.; Martín-Vallejo, F.J. Immediate effects of bilateral manipulation of talocrural joints on standing stability in healthy subjects. Man. Ther. 2009, 14, 75-80. [CrossRef] [PubMed]

8. Scheibel, A.; Debusschere, M. Changes in posture induced by 2 osteopathic manipulations are coherent with the sagittal or frontal orientation they involve. Agressologie 1991, 32, 134-136. [PubMed]

9. Chila, A.G. Fondamenti di Medicina Osteopatica; Casa Editrice Ambrosiana: Milan, Italy, 2015.

10. Hausdorff, J.M.; Nelson, M.E.; Kaliton, D.; Layne, J.E.; Bernstein, M.J.; Nuernberger, A.; Singh, M.A. Etiology and modification of gait instability in older adults: A randomized controlled trial of exercise. J. Appl. Physiol. 2001, 90, 2117-2129. [PubMed]

11. Alexander, N.B. Gait disorders in older adults. J. Am. Geriatr. Soc. 1996, 44, 434-451. [CrossRef] [PubMed]

12. Korr, I.M. Osteopathic research: The needed paradigm shift. J. Am. Osteopath. Assoc. 1991, 91, $156-161$. [PubMed]

13. Bankoff, A.D.P.; Bekedorf, R.G.; Schmidt, A.; Ciol, P.; Zanai, C.A. Anàlise do equilibrio corporal estàticoatravés de um baropodômetro eletrônico. Rev. Conexões. 2006, 4, 19-29.

14. Nakagawa, H.; Ohashi, N.; Watanabe, Y.; Mizukoshi, K. The contribution of proprioception to posture control in normal subjects. Acta Otolaryngol. Suppl. 1993, 504, 112-116. [CrossRef] [PubMed]

15. Baylor, A. Plasticity and exercise effects on aging motor function. In Development of Posture and Gait Across the Lifetime; Woollacott, M.H., Shumway-Cook, A., Eds.; University of South Carolina Press: Columbia, SC, USA, 1989; pp. 176-201.

16. Franchignoni, F.P.; Vanni, G.; Savoini, C. Computerized analysis of the effects of age on posture control. Minerva Med. 1985, 76, 835-839. [PubMed]

17. Fragkaki, I.; Stins, J.; Roelofs, K.; Jongedijk, R.A.; Hagenaars, M.A. Tonic immobility differentiates stress responses in PTSD. Brain Behav. 2016, 23, e00546. [CrossRef] [PubMed] 